\title{
Limiting Athletic Performance or Prohibiting Athletic Participation for Health Reasons: A Medical Ethics Viewpoint
}

\author{
Steven D. Stovitz \\ University of Minnesota
}

Our society generally views physicians and other medical personnel with respect. This respect is a result of not only their expertise in health issues but also the public's faith that physicians and other medical personnel use their expertise in an ethical manner. "Medicine is, at its center, a moral enterprise grounded in a covenant of trust" (Crawshaw et al., 1995).

To understand the role of the team physician in either preventing athletes from using banned substances and/or disqualifying student-athletes (SAs) from participation, it is essential to appreciate the similarities and differences in the application of ethical principles between physicians in sports medicine and those in standard medical practice. For this article, the term sports medicine physician refers to a physician who assumes the role of the team physician (i.e., it does not include someone who practices sports medicine but has no ties to the athlete's university). At the intercollegiate level, universities often employ team physicians. A physician employed in the role of team physician at a university accepts responsibilities to the team and university that fundamentally alter the standard doctor-patient relationship. While balancing these potentially competing loyalties, sports medicine physicians must seek to maintain the trust of SAs, the universities they work for, and society. Without trust, the sports medicine profession will lose power both as a confidant in the doctor-patient relationship and as a valued arbiter in intercollegiate sports.

Consider the following: A freshman football lineman comes in for his preparticipation sports physical and admits to using the new performance enhancing substance, "makemepro." This substance is believed to enhance muscle strength. There have been several anecdotal reports of cerebrovascular accidents (i.e., strokes) in users of this substance. It is believed that episodes of temporary blindness may be a warning sign for the drug-associated strokes. The drug is banned, but it is not detectable by current testing methods. The player informs you that he was a mediocre player before taking "makemepro." Now he is a high school All-

The author is with the University of Minnesota Medical School, Department of Family Medicine and Community Health, 420 Delaware Street SE, MMC 381, Minneapolis, MN 55455. 
American and is coming to your university on a scholarship. Without the scholarship, he would have no way of affording any school's tuition and thus would not attend college. He informs you that since starting "makemepro," he has short episodes in which he loses his vision, and he wonders whether these episodes are related to use of the drug. In addition, because he is certain that his performance on the football field is dependent on this drug, he tells you that there is no way that he will stop taking it. Knowing that the substance is banned, he asks that you do not tell the coach and administration. What do you do?

Given that universities entrust team physicians with preparticipation evaluation and that the major objectives of these evaluations include screening for lifethreatening issues and meeting medico-legal requirements, it is apparent that this physician cannot clear the athlete. Were the SA to suffer a stroke, the school could be at enormous financial risk. In addition, because the substance is "banned," the athlete's use of it could put the team's athletic standing in jeopardy. Were the physician in the private community, he or she could inform the athlete that clearance for participation cannot be granted, and further details could be handled in a confidential discussion between the doctor and the athlete, a competent adult (this pertains to college athletes, who are nearly always over 18 years of age). However, given that the school has entrusted this particular physician to protect its interests, it is incumbent on the physician to inform the SA that he cannot keep this information confidential.

Nonmedical personnel should understand that refusing to keep this information confidential contradicts a basic principle of classic medical ethics: patient autonomy. A fundamental element of the standard doctor-patient relationship is the ability of competent adults to make their own autonomous health care decisions (i.e., patient autonomy). The bioethical principle of patient autonomy underlies much of the standard of care for issues involving patient confidentiality, patient choice, and informed consent (Beauchamp \& Walters, 1994). In the classic doctor-patient relationship, the physician in the example would refuse the athlete clearance for full participation because the athlete has admitted to something that is potentially life threatening). The physician would not be expected to go against the wishes of the SA and call the school to inform them that the SA is using a banned substance. Indeed, physicians may only breach confidentiality in rare instances such as mandatory reporting laws (e.g., in cases of suspected child abuse) or imminent danger. There is no exception to confidentiality for community-based adult patients who cheat or take risks.

Whenever possible, community physicians should avoid serving in the role of law enforcement. For those who disagree, assume that you had a patient who informed you that over the past few months he had twice driven his car home from a bar while under the influence of alcohol, but he did not want you to tell his spouse or the police. It is fair to say that most physicians would keep this information private, perhaps choosing to counsel the patient on the hazards of drunk driving with the hope of preventing further episodes. Now, assume that the risk for a "makemepro"-induced stroke is lower than the risk of serious injury from drunk driving. Why couldn't an adult (i.e., the athlete) make a choice that gives him what he views as a benefit (in this case more strength, a college scholarship, and the chance to become a rich professional athlete) and accept the risks involved? Following the same logic as that for a patient who admits to occasionally driving 
under the influence of alcohol (where the risk/benefit ratio is much higher, especially given the lack of any large benefits), it follows that a physician would then counsel and follow the patient.

However, because of sports medicine providers' competing loyalties (again, where the physician is acting on behalf of a university or a governing body), the scope of patient autonomy is not nearly so broad. This highlights a major difference in the application of bioethical principles to typical medicine as compared with sports medicine. When a sports medicine physician is fulfilling an official role for a university (as in the case of high-level intercollegiate athletics), that physician has responsibilities to both the athletes and the school. In the case above, the physician's responsibility to the school calls for him or her to inform the student that the information cannot be kept confidential for the reasons outlined above. As suggested by Bernstein, Perlis, and Bartolozzi (2004), if an athlete reveals past medical history pertinent to his or her ability to safely participate, then irrespective of the athlete's wishes, the physician may and sometimes must break confidentiality by informing the team or school administration.

So, how does a sports medicine physician maintain the trust of SAs given the possibility of the "makemepro" scenario and others like it? Preparation and disclosure. As much as possible, before an SA's first encounter with the medical staff, physicians should disclose to SAs that because of obligations to the team and university, team physicians cannot guarantee confidentiality at all times. By informing SAs in advance that, unlike a standard physician-patient relationship, confidentiality might not be guaranteed, the physician discloses his or her obligations and minimizes the dilemma. (Pearsall, Kovaleski, \& Madanagopal, 2005; Waddington \& Roderick, 2002). If the SA has an established relationship with a hometown doctor, then it is often advisable to keep this relationship going in the event of the SA wanting to be more certain of confidentiality. After expressing an understanding of this, the SA can decide whether to tell the team physician certain pieces of information.

Critics of this approach might argue that such warnings might ultimately prove dangerous for athletes. That is, telling athletes that information might be forwarded to the coach or University could result in the dangerous underdisclosure of pertinent medical history. For example, many athletes might be reluctant to reveal a history of concussions if aware that it could bar them from competing. Not knowing which facts might be revealed to the coach, athletes could withhold sensitive or embarrassing information that might be very relevant to their general health.

Acknowledging this tradeoff, it is important to understand the role of trust in any doctor-patient relationship. Respect for SAs as persons supercedes the risks of underdisclosure and ultimately supports a trusting physician-athlete relationship. Unexpectedly breaking patient/athlete confidentiality can be most unprofessional and so damaging to the physician-athlete relationship, and subsequently to the physician-team relationship, that professionalism demands setting clear expectations. To prevent underdisclosure in other clinical settings it is important to explain that this limit of confidentiality is unique to the role of the physician covering the team and does not extend into the athlete's private clinical encounters. 
Patient autonomy is also limited in the sports medicine setting as a result of limits on patient choice. As discussed by Prof. Mitten, the case of Knapp v. Northwestern highlights this issue. Mr. Knapp accepted the risk of playing basketball given his cardiac condition. Although there were some medical experts who determined that the risks of participation were acceptable, the team physicians felt otherwise. The uncomfortable situation of prohibiting a SA from participating is within the jurisdiction of anyone who assumes the role of a university team physician. If a SA has a sign or symptom that the physician deems to pose an excessive risk to the university, then that physician has a duty to prevent the SA from participating. Whether the SA has a "right" to play college sports is a legal and philosophical question. From a medical standpoint, the physician must use his or her own judgment as a representative of the university. As protectors of the University, the team physicians had the right to deny Mr. Knapp the ability to play basketball at Northwestern. This does not mean that physicians need to prohibit athletes from playing on a frequent basis. On the contrary, the role of the sports medicine physician can be viewed as one that seeks to allow safe participation.

On a much more mundane level, the difference in the scope of patient choice between sports medicine and more typical medicine is played out on a regular basis. Imagine that a community physician sees a 20 -year-old female patient with a tibial stress fracture, and the physician recommends no running until a reassessment in three weeks. The SA returns in 3 weeks with continued pain and the physician learns that she had been running long distances on a daily basis against medical advice. The physician would document this and inform the SA of the risk of continuing to run on a stress fracture. Beyond that, the physician would not be expected to inform others or take any extra means to limit the choice of a competent adult.

Now, imagine that this same patient was a scholarship cross-country runner at a university, and she was seeing the team physician. Here, we once again see the more limited role of confidentiality, because the team physician has the right and responsibility to discuss this issue with her coaches and other athletic staff personnel. This could lead to steps that would limit the SA's options for running, or at a minimum, add further downsides to the choice of continued running (e.g., loss of scholarship). The SA has a choice as to whether to be on the team. If she chooses to be on the team, she accepts the rules of the team. One of these rules might be to follow medical advice.

Although the issues of confidentiality and patient choice limit the amount of patient autonomy in university-related sports medicine encounters, the issue of informed consent must not. Here, sports medicine physicians must uphold the same standards of consent as their community counterparts. The necessary conditions for legitimate, informed consent in the field of medicine (including sports medicine) are as follows:

- Disclosure: All relevant information that a reasonable person in this player's position would want to know regarding the proposed treatment, nontreatment, and alternative treatments must be explained to the player in an appropriate fashion.

- Capacity: Players must have the capacity to understand this information and appreciate how it applies to them. "Competency" is the legal system's equivalent to the medical term "capacity." 
- Voluntariness: Within a noncoercive environment, the player must voluntarily express his or her wishes regarding the proposed options.

Certainly the issue of coercion, given the pressures upon any member of a team to conform, poses a potential challenge to fulfilling the condition of voluntariness. Still, maintaining these standards as close to ideal as possible is smart not only medically but also legally. Proper informed consent forms the basis of the best medical-legal defense for the sports medicine physician. Athletes are constantly making judgments that could hamper their long-term health, and the physician covering the team is often involved in those decisions.

What about the harm that athletics itself causes the body? There is a strong medical argument that playing football while carrying excessive weight on internally deranged knees accelerates osteoarthritis. Why is it appropriate that physicians allow athletes to damage their bodies through sports participation but not through the use of banned substances? The risks associated with the sports themselves are assumed to be known and accepted by the athlete. When athletes are brought into a sports medicine encounter (e.g., with an injury), it is important that the physicians inform the athletes of the risk of further play (beyond the baseline risks of the sport) and allow the athlete to decide. The fact that team physicians are not successfully sued more often is based partly on the fact that the adult athlete was appropriately informed of, understood, and voluntarily accepted these medical risks. Unlike the issue of confidentiality, the scope of informed consent is similar in the ethics of sports medicine and the ethics of typical medicine. It is essential that sports medicine physicians resist any temptation to deviate from the standards of informed consent as it is routinely practiced.

The issue of the sports medicine physician's role in athletes' use of performance-enhancing substances also involves the ethics of patient autonomy. Sports medicine physicians must be diligent in avoiding the active dispensation or encouragement of banned substances. Here, the application of ethical principles are generally similar to the those in regular medical practice. Respecting patient autonomy means respecting the choices the patient makes, not necessarily facilitating anything they want to do.

Imagine that a SA came with his coach (for the sake of drama, make it the new football coach who recently signed a multimillion dollar contract and is generally hailed as the answer to all of the school's problems) and asked a physician to assist them in obtaining "makemepro." The physician would not be expected to assist in obtaining this banned drug. The exact criteria that makes one substance banned and another an accepted performance-enhancing substance is a matter of debate and not a focus of this manuscript. Disregarding the issue of whether the substance is banned, the situation is similar to other situations physicians frequently encounter in which an adult requests something for a quality-of-life benefit that might carry hazardous side effects. Here the crucial issue is that the substance is banned, and not that it carries some risk.

Consider the issue of medications for erectile dysfunction. These carry rare but serious side effects. Yet, they are commonly prescribed because adult patients consider the benefits to outweigh the risks. With SAs and the drugs for enhanced performance (athletic performance, that is), the issue is that the substances are banned and thus all physicians (i.e., the team physician or a community physician) must avoid ever knowingly providing any SA with a banned performance- 
enhancing substance (in this case, "makemepro"). Given the frequency with which the rules of banned substances change and the lack of regulation regarding supplements, physicians are wise to try to avoid directly prescribing or encouraging use of any substances that claim to enhance performance.

Note that in this latter example, there is a possibility that a physician working for a team might have a motive that makes the team physician, in fact, more likely than the community physician to assist the athlete with obtaining the drug. This might be the case if the physician valued his or her team physician role ahead of his or her societal role as a beholder for the integrity of sports. Although hoping that one's team wins is a natural response to a game, when physicians become fans, investors, or simply rest their pride on serving as team physicians, they can lose the impartiality necessary for optimal judgment. Emotional ties to teams gain appeal as the level of competition rises from high school through college and to the professional level (Polsky, 1998). Athletic department administrators and coaches must avoid asking their team physicians to act in ways that might compromise the physician's role as a source of public trust, and beware of physicians who have a reputation of breeching their ethical responsibilities.

In summary, to understand the issues that sports medicine physicians face when banning SAs from participation and/or discouraging pharmaceutical performance enhancement, one must look beyond the conventional clinical-ethics literature. It is important to understand the similarities and differences in the application of ethical principles to sports medicine as compared with regular medical practice. The two are similar in the need to fully inform SAs of their treatment options. Differences arise because physicians are responsible not only to their patients (here, the SAs) but also to the university. Consequently, patient autonomy might be limited in some respects as a result of the organizational structure surrounding the physician-SA relationship. The scope of patient confidentiality and the freedom with which patients may choose to go against medical advice are more limited within this relationship. SAs and universities need to understand the competing loyalties facing physicians in sports medicine settings. Sports medicine physicians need to engage in ethical behavior that garners respect. The key is to maintain the trust of both the SAs and the universities and, consequently, the trust of the society-at-large. In this manner sports medicine physicians can maintain their esteemed role, and SAs can continue to reap the benefits of safe participation in intercollegiate sports.

\section{Acknowledgment}

The author wishes to acknowledge David Satin, MD, an assistant professor in the University of Minnesota's Department of Family Medicine and Community Health and a post-doctoral fellow at the University's Center for Bioethics for his comments on this manuscript.

\section{Reference List}

Beauchamp, T., \& Walters, L. (1994). Contemporary issues in bioethics (4th ed., pp. 2223). Belmont, CA: Wadsworth.

Bernstein, J., Perlis, C., \& Bartolozzi, A.R. (2004). Normative ethics in sports medicine. Clinical Orthopaedics and Related Research, 309-318. 
Crawshaw, R., Rogers, D.E., Pelligrino, E.D., Bulger, R.J., Lundberg, G.D., Bristow, L.R. (1995). Patient-physician covenant. Journal of the American Medical Association, 273, 1165-1266.

Pearsall, A.W., Kovaleski, J.E., \& Madanagopal, S.G. (2005). Medicolegal issues affecting sports medicine practitioners. Clinical Orthopaedics and Related Research, 50-57.

Polsky, S. (1998). Winning medicine: Professional sports team doctors' conflicts of interest. The Journal of Contemporary Health Law and Policy, 14, 503-529.

Waddington, I., \& Roderick, M. (2002). Management of medical confidentiality in English professional football clubs: Some ethical problems and issues. British Journal of Sports Medicine, 36, 118-123. 